\title{
LA TRANSFORMACIÓN URBANÍSTICA DE COMPOSTELA. EL PLANEAMIENTO URBANO AL SERVICIO DE UN PROYECTO DE DESARROLLO (GALICIA, ESPAÑA)
}

\author{
(THE URBAN TRANSFORMATION OF SANTIAGO DE COMPOSTELA. CITY PLANNING AT THE \\ SERVICE OF A DEVELOPMENT PROGRAMME. GALICIA, SPAIN)
}

\author{
Juan Luis Dalda Escudero, Anxel Viña Carregal \\ Codirectores del Plan General Municipal y del Plan Especial de Protección de la Ciudad Histórica de Santiago de \\ Compostela
}

ESPAÑA

Fecha de recepción: 30-VIII-95

\begin{abstract}
RESUMEN
El ambicioso proyecto urbanistico para la capital de la Comunidad Autónoma Gallega, que está siendo ejecutado con una eficacia sorprendente, articula su propuesta de ordenación para la ciudad sobre el objetivo principal de recuperar y salvaguardar su magnifico Conjunto Histórico. Imprescindible para contextualizar las diversas actuaciones que se presentan en estos dos números monográficos dedicados a Compostela, el articulo descubre los planteamientos fundamentales que permiten comprender el proyecto de renovación y modernización urbana que se está desarrollando en Santiago de Compostela desde la aprobación del Plan General del año 1989.
\end{abstract}

\section{SUMMARY}

The ambitious city planning for the capital of the Autonomous Community of Galicia, which is being carried out with outstanding efficacy, builds its proposals for the organisation of the city around the main objetive of recovering and safeguarding its magnificent Historic Area. This paper, essential in the context of the various planning actions presented in this two monographic volumes dedicated to Compostela, explains the fundamental approaches that allow the understanding of the urban renovation and modernisation projects now in course in Santiago de Compostela since the General Plan was approved in 1989.

\section{El contexto de la planificación y el horizonte del desarrollo urbano.}

Las obras y proyectos que se presentan en esta publicación están ligados por el nexo común de producirse como desarrollo de un proyecto urbano instrumentado a través de la planificación urbanística municipal vigente desde 1990.

Con el deficiente marco de un planeamiento urbanístico heredado de los años 70, la ciudad de Santiago de Compostela había alumbrado, en la década de los 80 , unas transformaciones urbanas que anunciaban la eclosión que se había de producir a partir de 1990. Dichas transformaciones se produjeron de la mano de procesos sociales, económicos y políticos que han incidido muy directamente en la ciudad, dando lugar a una evolución muy diferente a la del conjunto de las ciudades gallegas y aun españolas que se resentían durante aquellos años de los efectos de la crisis económica.

La población de hecho de Santiagoen 1986 es casi vez y media superior a la de 1970; en 1982el Parlamento Gallego convierte a la ciudad en sede de las instituciones autonómicas de Galicia, impulsando un renovado proceso de 
expansión y diversificación terciaria, mientras la Universidad de Santiago vivía una fase de crecimiento expansivo; en 1985 la Ciudad Histórica se incorpora al Catálogo del Patrimonio Mundial de la UNESCO. Las transformaciones urbanas inducidas por esta dinámica realidad se producían por el impulso autónomo de la acción directa de los agentes inmobiliarios y de las administraciones públicas sectoriales, sin que se hubiera alcanzado ningún tipo de intervención directiva y equilibradora de la administración urbanística, prácticamente, hasta la tramitación del planeamiento y la urbanización del Polígono Público de viviendas de Fontiñas (año 1986) y aún en este caso sin disponer de una reflexión técnicamente acabada sobre la ciudad en su conjunto, a lo que se añadiría, en fase de ejecución, la reelaboración autónoma y precipitada de la ordenación del polígono.

De este modo, el Plan General de 1989, se redacta en un momento en el que la ciudad se halla inmersa en un proceso de crecimiento urbano de características diferentes al conocido en las décadas anteriores. En esta ocasión, el crecimiento urbano, aun siendo cuantitativamente importante, lo es más desde el punto de vista cualitativo por cuanto está dando lugar a una diversificación de las actividades tradicionales, debido al enriquecimiento de los roles que la Ciudad desempeña a nivel comarcal, regional, nacional e internacional. El Plan de Ordenación Urbana que se redacta entre 1988 y 1989 trató de potenciar este impulso, articulando, coordinando e induciendo iniciativas al servicio de un proyecto de ciudad más integrada socialmente, con una economía diversificada y sólida, mejor dotada de servicios públicos, funcionalmente eficiente y en la que la armonía y belleza de una ciudad histórica recuperada y viva alcanzara a integrarse en un entorno, constituido por la Ciudad toda, de la debida calidad arquitectónica y urbanística.

Con tales antecedentes, se perfilaba una caracterización general de la ciudad que, en el horizonte de programación del Plan General, estaría marcada por los siguientes rasgos:

-Santiago se presenta como una ciudad de tamaño medio entre las gallegas, como un centro regional especializado y con fuerte presencia del terciario público. Formando parte del corredor litoral atlántico (Coruña-Vigo-Porto), su carácter nodal y su buena relación con la Galicia interior a nivel de comunicaciones y servicios la sitúa en una posición equilibradora y articuladora del sistema urbano gallego. Sus funciones simbólicas y representativas no hacen sino reforzar dicha posición.

-La ciudad se consolida como un importante centro terciario en el que destaca la fuerte presencia de la Universidad, cuyas perspectivas de expansión demandarán una ampliación de la infraestructura universitaria docente $y$, sobre todo, investigadoray de servicios, con un crecimiento, moderado en términos absolutos, de la matrícula.
-El perfil de especialización, muy marcado por el terciario público, se prevé pueda producir el encadenamiento de un terciario privado, más diversificado, aumentando el peso del comercio, los servicios de empresas, las comunicaciones y el transporte.

-La singular relevancia cultural de su patrimonio histórico permitía pensar en la consolidación de un movimiento turístico en expansión, que, en virtud de sus características, exigirá no sólo la ampliación y mejora de la infraestructura hotelera y hostelera, sino también el impulso de las actividades culturales, científicas y de esparcimiento, de comunicaciones y de transportes.

-Así pues, la ciudad presentaba unas perspectivas de desarrollo urbano en el cual, con ser relevante el crecimiento demográficoy su expresión residencial, vendría a resultar tanto o más significativo el efecto urbanístico de las actuaciones relacionadas con la acomodación de la estructura de la ciudad a los usos en expansión, que van configurando una ciudad abierta con gran capacidad de recepción y acogida.

-La concentración y diversificación de actividades y funciones exigen producir un salto cualitativo de la estructura urbana de la ciudad, que en ese momento se hallaba colapsada. La nueva estructura ha de dar respuesta a las demandas funcionales de los movimientos, tanto internos como de llenado y vaciamiento de una ciudad de acogida deficitaria en infraestructura viaria, condicionada fuertemente por las características propias de un tejido histórico central y radial. La nueva estructura que se

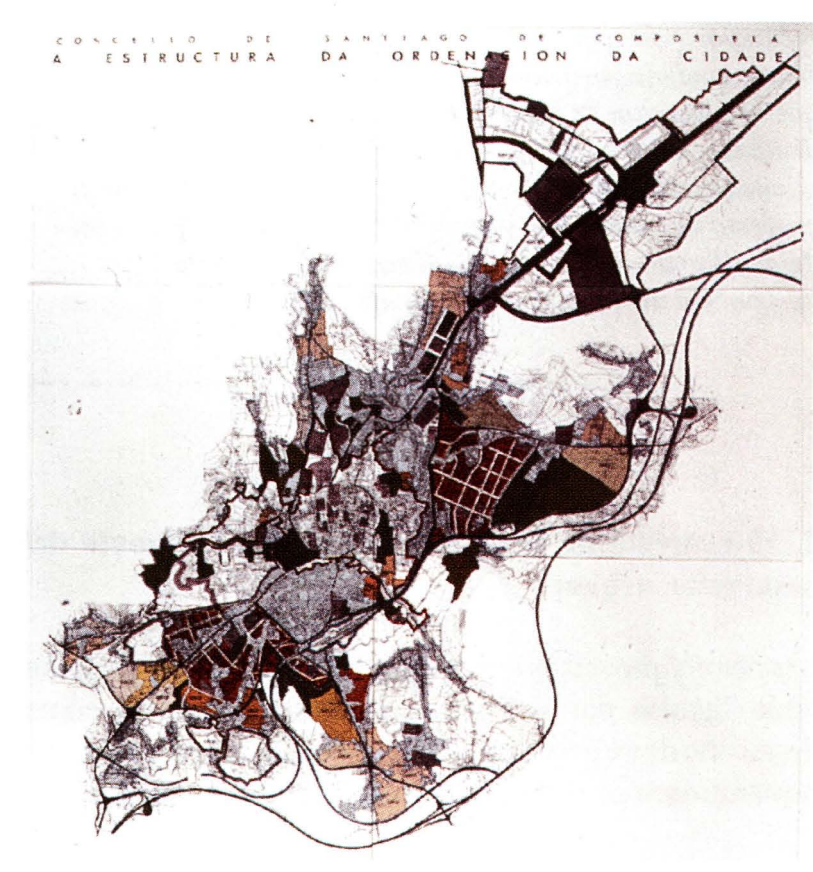

Plan General Municipal de Ordenación, 1989. Plano de la estructura general de la ordenación. 
propone procura el mantenimiento de una dimensión y compacidad del agregado urbano denso que permita unas relaciones con el centro, y muy especialmente con el Centro Histórico, con la menor dependencia del transporte privado, viable peatonalmente, produciendola localización, periférica y bien relacionada con el sistema de comunicaciones, de actuaciones de remate en las entradas de la ciudad vinculadas a los grandes usos direccionales (hospitalario, administrativo, ocio-recreativo, terciario privado, industrial, etc.).

La relación de equilibrio entre tráfico rodadoy peatonalidad ha exigido la definición pormenorizada del dimensionado y tratamiento urbano del nuevo sistema viario estructural que pende axilmente de la carretera de Circunvalación en su nueva traza larga, ahora reelaborada como gran colector urbano y urbanizado.

-La estructura urbana propuesta está concebida y orientada hacia la configuración de una realidad urbana acabada y continuaen sus estándares de urbanización y de urbanidad. En esta perspectiva, el equilibrio entre el centro y sus periferias (algunas muy inmediatas) permite otra lectura desde el prisma del proyecto urbanístico de la ciudad, con la oportunidad de elaborar las nuevas fachadas y puertas de una ciudad abierta radialmente en sus funciones de acogida.

-La concentración de los esfuerzos urbanizadores en el acabado de la ciudad existente y en la ordenación de las piezas yuxtapuestas de nueva urbanización (Fontiñas, San Lázaro, Zona Sur, Ponte Pedriña, ...), corregidas con las medidas de integración entre partes, hacen pensar en una ciudad compleja funcionalmente, debido a sus deficiencias estructurales, peromejor administrada en sus recursos infraestructurales, con mayor relación entre sus partes y más equilibrada en el nivel de urbanización y dotaciones.

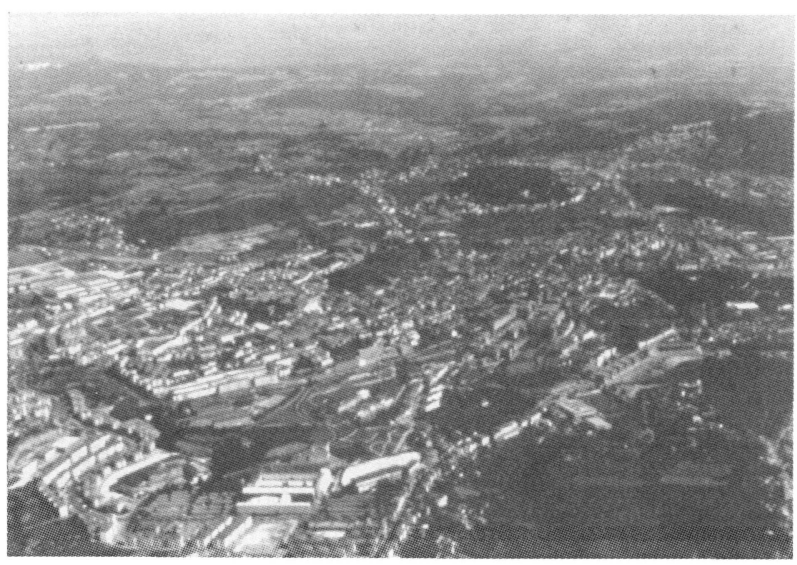

Vista aérea de la ciudad desde el Norte, 1992. (Fotografía Paisajes Españoles).

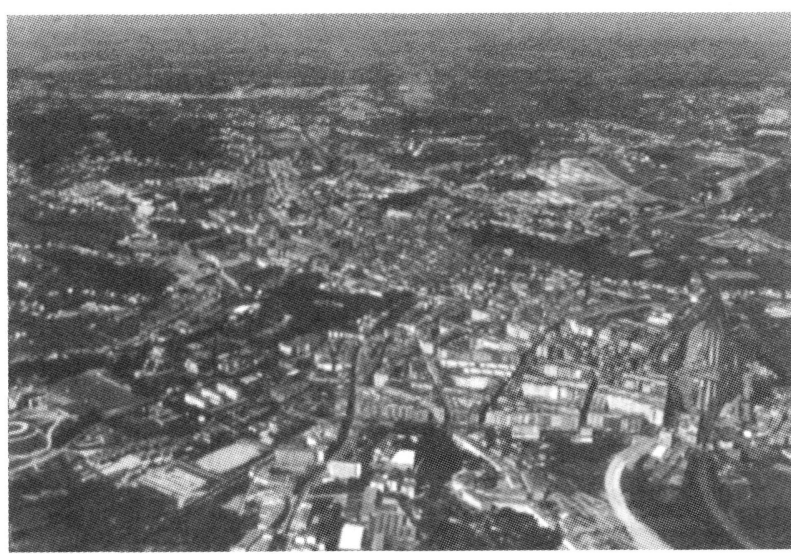

Vista aérea de la ciudad desde el Sur, 1992. (Fotografía Paisajes Españoles).

-El impulsoa las políticas de rehabilitación y de recuperación urbana y el esfuerzo disciplinar urbanístico y arquitectónico en la construcción de la ciudad de Santiago, podrían producir, en estas condiciones, una transformación positiva en la que la protección de los valores históricos en el marco de una recualificación del conjunto urbano haga recuperar el control sobre las condiciones físicas, funcionales y ambientales de una ciudad de la significación de Santiago.

Con esta perspectiva, la planificación urbanística se ha concebido como la primera de las palancas para impulsar un proceso de desarrollo de base urbana en el que la sucesión de hitos, eventos y conmemoraciones han servido de acicate y estímulo para el desencadenamiento de los efectos dinamizadores de un complejoy ambicioso proyecto urbano. Es así como, primero, con la celebración del Año Jacobeo 1993, ahora, en 1995, con la conmemoración del V Centenario de la creación de la Universidad de Santiago $\mathrm{y}$ en el futuro inmediato la celebración del Año Jacobeo 1999 y la Capitalidad Cultural Europea compartida en el año 2000, se van encadenando, como ocasiones para una transformación urbanística, pero también económica y social, con implicaciones que trascienden al ámbito 1ocal de una ciudad de la dimensión de Compostela.

\section{El concepto básico de la ordenación urbanística}

El proceso de formación del agregado urbano de Santiago de Compostela ha dado lugar a la configuración de una ciudad de forma arriñonada, ceñida a las laderas del Pedroso y al cauce del Sarela, recrecida en la dirección del Camiño Francés con la nueva implantación de Fontiñas y rematada, en la propuesta de este Plan, con la implantación terciaria, deportiva y recreativa de Ponte S. Lázaro. (Estadio Municipal, Palacio de Congresos, etc.). 
La ciudad se conforma en un crecimiento dominado por una directriz Nordeste-Suroeste. La estructura resultante no puede materialmente configurarse como una estructura mallada y tampoco concéntrica y tiende a presentarse como un conjunto de piezas relativamentecerradas, colgadas de los ejes viarios que conducen al centro histórico y ocupando los espacios interejes. Estas piezas estaban en contacto con el recinto amurallado hasta la aparición de los barrios de vivienda pública de Vite y Pontepedriña y es a partir de ese momento cuando pasan a producirse en una segunda corona, a la que se añaden ahora Fontiñas, Conxo, Cornes y Choupana. $\mathrm{Si}$, en relación a la primera corona de crecimiento, el Centro Histórico y su infraestructura viaria actuó de nexo de conexión y referente central, la consolidación de la segunda corona ha requerido de un enriquecimiento del sistema urbano que obliga ahora a plantearse el reequilibrio de usos en la ciudad y la integración plena del distribuidor constituido por la actual circunvalación.

Partiendo de esta estructura urbana, el Plan General de 1989 se propone compatibilizar la centralidad urbana del continuo CascoHistórico-Ensanche que concentra servicios, comercioy actividades urbanas de todo tipo-con la densidad propia del lugar central de un agregado urbano que se mantiene en una escala de peatonalidad- con la descentralización de servicios, dotaciones y comercio cotidiano, mediante un esfuerzo por equiparar el nivel de infraestructuras urbanas de las periferias con el del centro. Adicionalmente, opta por ladescentralización de los grandes usos generadores de direccionalidad (servicios administrativos nacionales, comercio regional público y privado, ocio-recreativo regional, dotacional extensivo, sanitario regional) de modo tal que se consiga

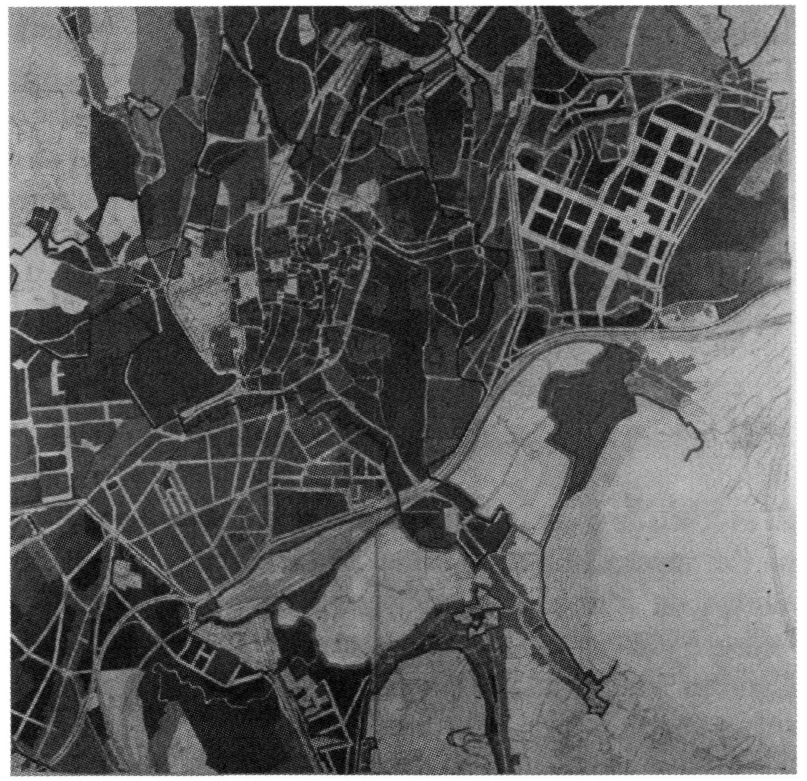

Plan General Municipal de Ordenación, 1989. Detalle de la ordenación. descongestionar el centrourbano parafacilitar sus funciones comerciales y de relación, al tiempo que se difunde un cierto tipo de urbanidad en toda la periferia; que habrá de favorecer la integración de losbarrios periféricos al complejo sistema urbano de la ciudad terciaria.

La propuesta de conjunto trata de aprovechar los efectos positivos de la compacidad y continuidad de la ciudad para mejorar la relación entre sus partes y con el Centro Histórico, así como para difundir la actividad urbana en todo el conjunto. Para ello se produce el remate y acabado de la ciudad expandida en los últimos treinta años mediante: la conclusión de los espacios intersticiales todavía vacantes reservados, en general, para dotaciones o espacios libres; el tratamiento continuo de la urbanización en todo el conjunto; el remate de los bordes exteriores con propuestas vinculadas a la localización del sistema gran terciario con la intención formal de que sirvan a la formalización de las nuevas fachadas urbanas; el tratamiento urbano de los sistemas viarios de acceso, distribución y vías de borde y de sus nudos urbanos, y la conclusión de la dotación de equipamientos de la ciudad consolidada.

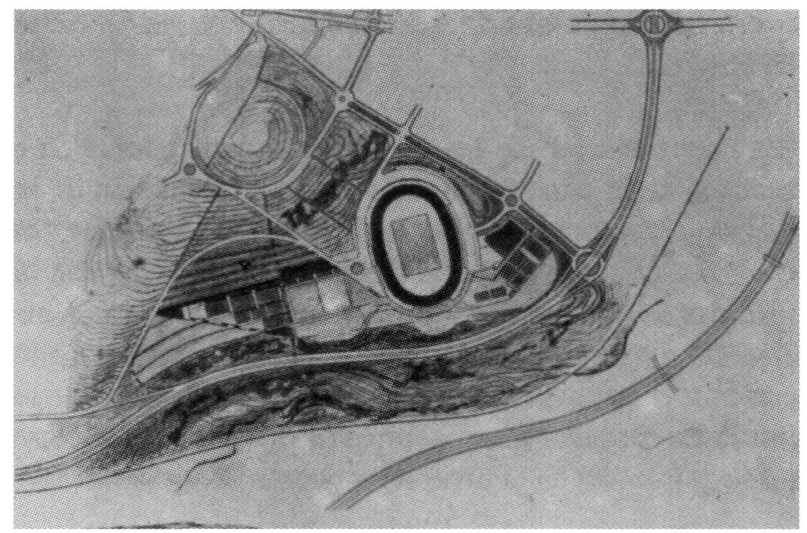

Plan Especial de Ordenación del Área Deportiva de San Lázaro, 1990.

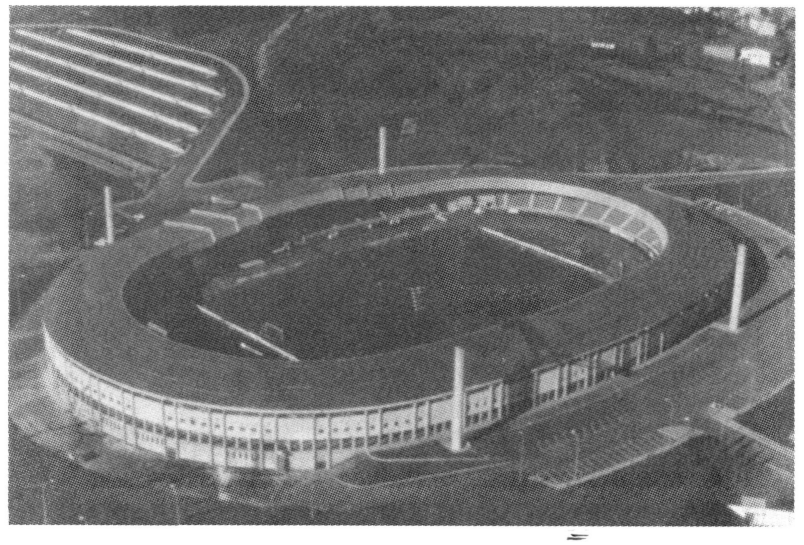

Estadio Municipal de San Lázaro. (Arquitecto: Andrés Fdez-Albalat Lois). 
3. La Ciudad Histórica como eje de la propuesta urbanística del Plan General: una acción global de recuperación urbana como marco para su protección y rehabilitación.

Por primera vez en la historia del planeamiento urbanístico de la ciudad, su conjunto histórico es objeto primordial de la reflexión urbanística, y su recuperación se convierte en objetivo principal en torno al cual gira la propuesta de ordenación de la ciudad.

Las propuestas del sistema general de comunicaciones, la localización de los grandes usos, la previsión de las áreas de crecimiento, el diseño del sistema de dotaciones públicas y la regulación de la ordenación del territorio municipal, obedecen al criterio general de propiciar una estructura urbana compacta, con una organización de usos que alivie la congestión de las zonas centrales y dimensionada proporcionadamente en relación a la Ciudad Histórica en una escala todavía peatonal, de modo que permita a esta parte de la ciudad mantener y reforzar las funciones urbanas que la mantienen viva.

El desarrollo simultáneo y bajo una dirección común de los trabajos del Plan General Municipal y del Plan Especial de la Ciudad Histórica ha permitido desarrollar estrategias de conjunto, con incidencia en la estructura general de la ciudad y en las políticas urbanísticas sectoriales, imprescindibles para dar solución a los problemas de la ciudad histórica.

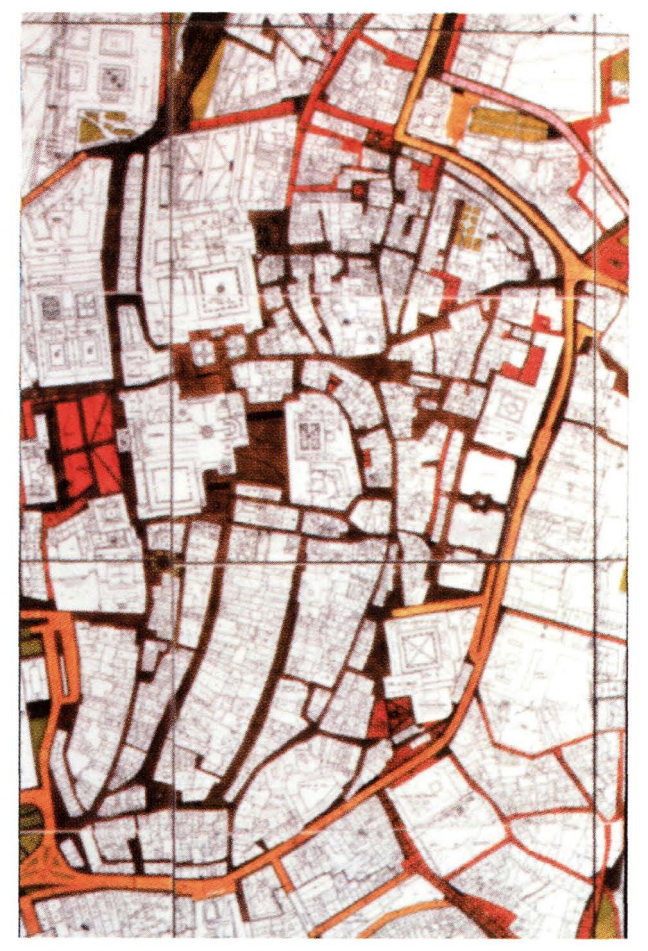

Plan Especial de Protección y Rehabilitación de la Ciudad Histórica. Plano de ordenación de la red viaria.

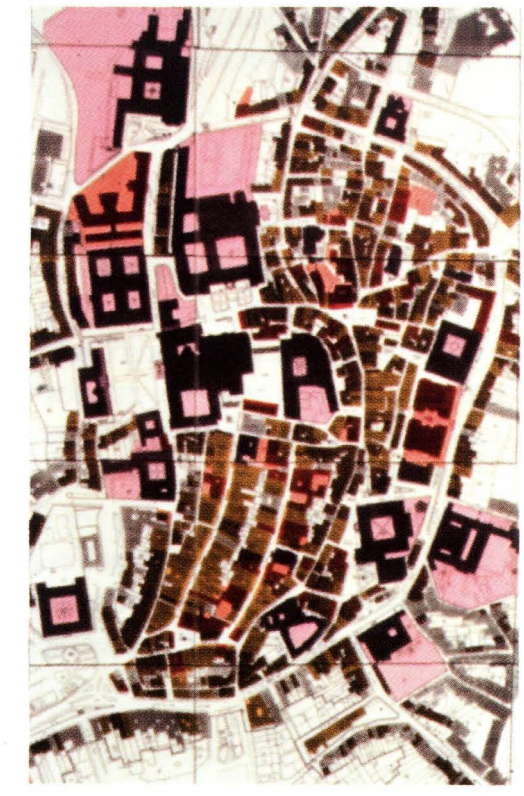

Plan Especial de Protección y Rehabilitación de la Ciudad Histórica. Plano de catalogación del patrimonio construido.

La puesta en marcha del Plan General a partir de 1990 permitió el desencadenamiento de una sucesión de medidas de gran relevancia para hacer posible la política de recuperación de la Ciudad Histórica. Estas medidas se refieren a los elementos básicos de la estructura urbana con incidencia en la problemáticaurbanística del tejido histórico.

-El desarrollo, ejecutado ya en su práctica totalidad, de las infraestructuras básicas de comunicación de la Ciudad permitirá descargar de tráfico de paso el sistema interior y, muy especialmente, la vieja ronda exterior a la muralla (antigua carretera $\mathrm{N}-550$ ) que congestiona su funcionalidad interior y segrega los rueiros históricos en su conexión peatonal con la Almendra central.

-Buena parte de las actuaciones públicas de implantación de los grandes servicios, equipamientos y dotaciones de la ciudad, inciden en la recuperación urbanística y ambiental de la fachada Oeste de la Ciudad Histórica, en la que la presencia monumental de la ciudad mantiene con plena vigencia su diálogo con el entorno rural. El traslado del Hospital General de Galicia, en fase avanzada de construcción, hace desaparecer el mayor generador de tráfico direccional y de demanda de aparcamiento de la zona, aliviando la congestión de O Pombal-Campo da Estrela, evitando la entrada de importante tráfico externo en el sistema viario urbano.

El traslado del Estadio Municipal a San Lázaro, además de incidir en el mismo aspecto funcional, permite la reelaboración de toda la ribera del río Sarela, recuperada 
para usos públicos deportivos extensivos, permitiendo aminorar el impacto de las viejas instalaciones deportivas de Sta. Isabel.

Las actuaciones universitarias en OBurgo das Nacións, en el Campus Sur y en la finca Simeón, aceleradas con motivo de la celebración del V Centenario de la Universidad de Compostela, contribuyen a consolidar la propuesta de ordenación para la mejora de la fachada Oeste mediante la presencia de usos universitarios intercalados con los tejidos residenciales ya existentes y los nuevos propuestos, de tal modo que se enriquezca su vida urbana con usos generadores de tráficos peatonales y menos dependientes del vehículo privado, preservando la relación de la Ciudad Histórica con el Monte Pedroso, y desechando, por tanto, la idea de cerrar la Ronda de Circunvalación por sus laderas.

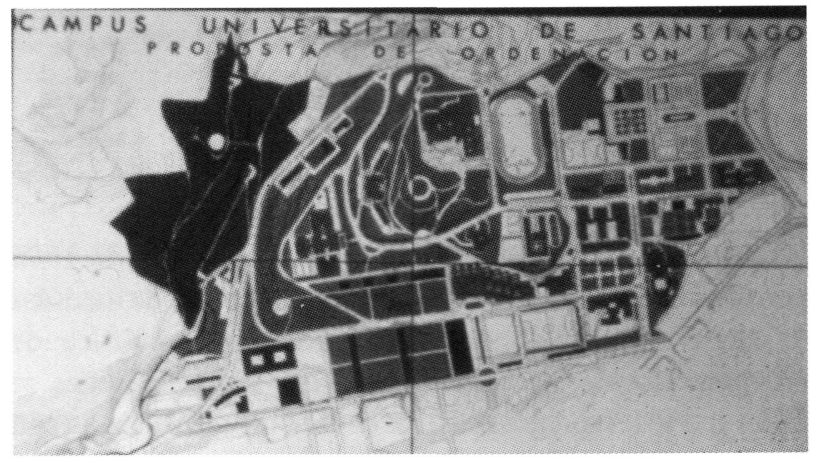

Plan Especial de Ordenación del Campus Universitario, 1991.

-Entre las actuaciones de nueva urbanización previstas por el Plan General se encuentran: la conclusión del Plan Parcial de Fontiñas público y privado y la urbanización de los polígonos que restan de los Planes Parciales de Sar y Almáciga.

La reciente entrada en funcionamiento del nuevo barrio de Fontiñas, colgado del Camiño Francés y de Os Concheiros, tendrá una gran incidencia sobre la importante pieza del tejido histórico formada por Belvís, A Trisca, rúa do Medio y rúa de San Pedro.

A partir de la implantación de una población en torno a los 15.000 habitantes, la vía histórica de entrada a la ciudad cobra nueva importancia, ahora como eje urbano de acceso peatonal al Centro Histórico. Esta oportunidad hará posible la mejor integración urbana de unos barrios históricos que, hasta el momento, ocupaban una posición excéntrica y terminal, dando sentido a la recuperación urbana del recorrido rúa de San Pedro-Porta do Camiño-Casas Reais, el Camino por excelencia.
La conclusión del Plan Parcial de Sar permitirá acabar el ligamiento del rueiro de Sar con el Ensanche, con ello, además de mejorar la integración urbana del rueiro histórico, aproximándolo a una zona de servicios y dotaciones de los que carece, se enriquece el viario urbano con lo que será posible disminuir la presión del tráfico rodado en beneficio del tráfico peatonal y de la vida urbana del barrio.

La actuación de remate del Monte da Almáciga cumple parecida función. Restablece la relación de los nuevos barrios de rúa do Home Santo y rúa de Betanzos, con la Almáciga e integra los ensanches pobres del Nordeste con la Ciudad Histórica, a través de la gran operación dotacional de Santo Domingo de Bonaval con el Centro de Arte Contemporáneo y su Parque, confluyendo así una serie de acciones en la revalorización del espacio simbólico de A Porta do Camiño.

-Desde las primeras reflexiones en torno a las propuestas del planeamiento urbanístico, existe conciencia clara de que la posibilidad de impulsar una acción generalizada de rehabilitación de la vivienda en la Ciudad Histórica depende de la desactivación del grave proceso de especulación inmobiliaria que sufrió la ciudad en la pasada década. Las expectativas generadas por este proceso han influido también en el tejido histórico, poniendo en riesgo la pervivencia de la sustancia tipológica en el caserío mayor y fomentando el abandono y deterioro del caserío menor.

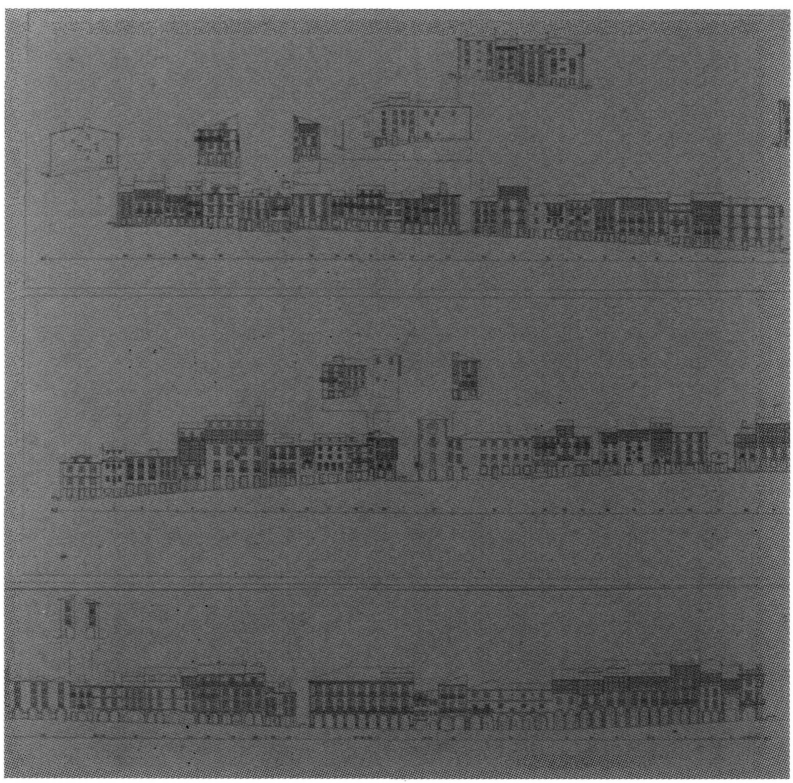

Plan Especial de Protección y Rehabilitación dêta Ciudad Histórica. Detalle de alzados. 
La rehabilitación de viviendas, con la preservación de los edificios y la recuperación residencial de un conjunto de tal tamaño, sólo sería posible en unas condiciones de mercado normalizadas en las que las políticas de ayuda y fomento puedan cumplir su doble función de: apoyo financiero a la vivienda para los sectores sociales que requieren de dichas ayudas y canalización de recursos públicos para preservar el patrimonio cultural, en este caso arquitectónico.

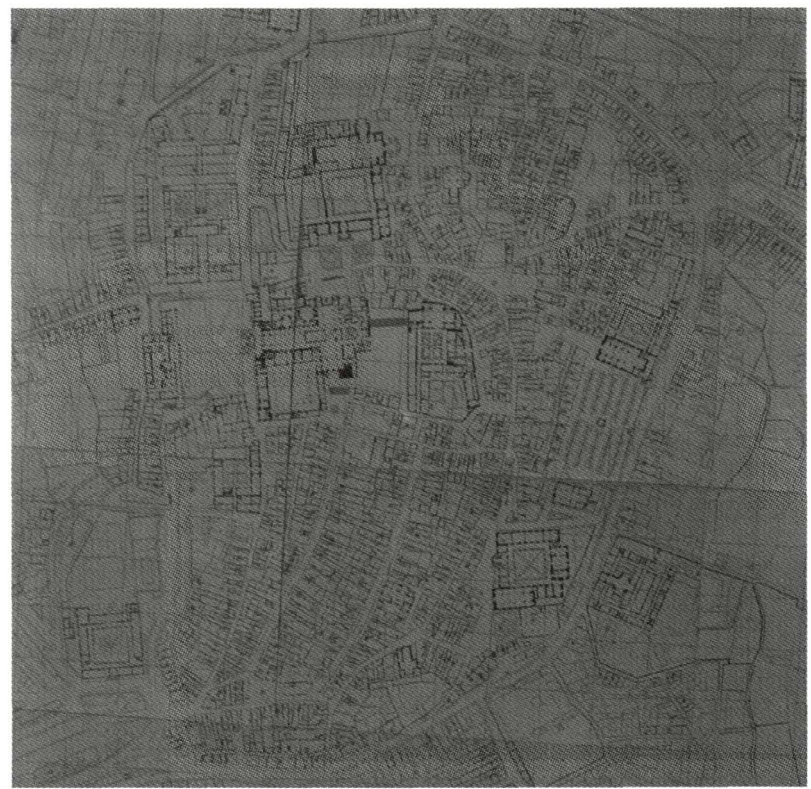

Plan Especial de Protección y Rehabilitación de la Ciudad Histórica. Planta tipológica. Detalle.

La creación de estas condiciones depende del éxito de la política general de suelo y vivienda en el conjunto de la ciudad. A tal efecto se ha establecido un programa dimensionado y con asignación concreta de recursos y operadores que garantice la presencia en el mercado de una oferta significativa de viviendas a precio tasado en los distintos regímenes de protección oficial. La manifestación de los primeros efectos de estas actuaciones ha sido condición previa para el impulso, a partir de 1994, de la acción extensiva de la rehabilitación de vivienda en la Ciudad Histórica.

La delimitación del ámbito del Planeamiento Especial de la Ciudad Histórica duplica el actual perímetro morado de protección. Con ello se extiende la protección a los tejidos urbanos históricos de los rueiros populares, que podrán ser rehabilitados e integrados en la ciudad central. La mayor extensión de lo ahora delimitado como Ciudad Histórica, su nueva funcionalidad y estructura en el conjunto, ha permitido, la identificación de piezas vacantes englobadas entre los tejidos edificados, así como otras áreas de oportunidad, abocadas a la intervención urbanística. El

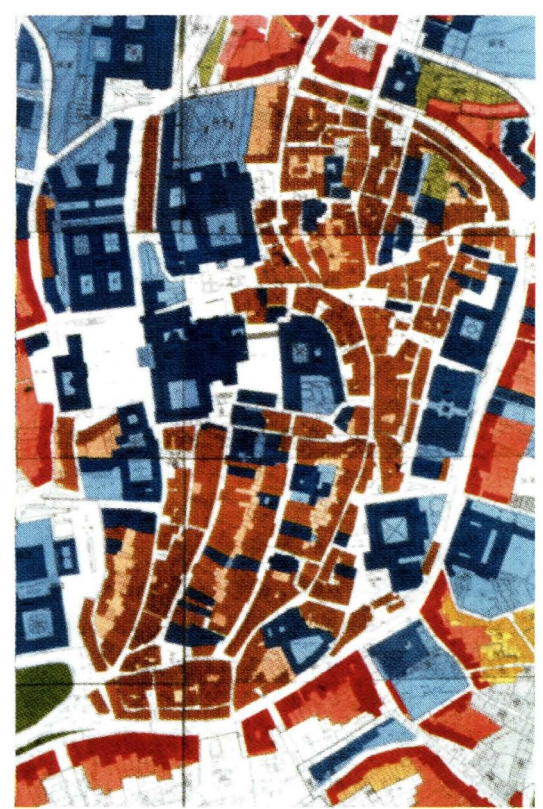

Plan Especial de Protección y Rehabilitación de la Ciudad Histórica. Plano de calificación.

Plan General fijó sus ámbitos y características funcionales en relación a la ordenación de la ciudad, estableciendo las determinaciones y parámetros genéricos de implantación. La intervención en estas áreas, vinculada a la idea de acabado y compleción de la ciudad, se ha definido pormenorizadamente en el Plan Especial de la Ciudad Histórica.

El encadenamiento del proyecto urbano, desde la escala de la planificación general hasta la del proyecto arquitectónico oinfraestructural, pasando por la ordenación urbanística de escala intermedia, constituye una de las características más singulares de la transformación urbanística que vive en estos últimos años la ciudad de Santiago de Compostela.

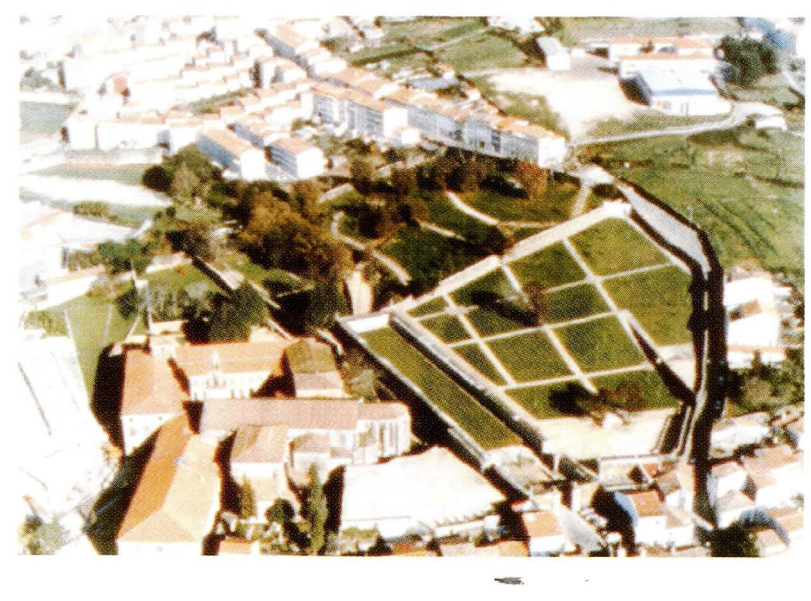

Centro Galego de Arte Contemporáneo. (Arquitecto: Alvaro Siza Vieira). 
La contextualización de actuaciones tan diversas como las que ahora se presentan en esta publicación, en el marco de un proyecto urbanístico complejo, y la fidelidad a la idea de potenciar el desarrollo de la ciudad, acrecentandola cualidad urbanística y arquitectónica como el principal y más singular valor de Compostela, son los rasgos que caracterizan esta dinámica fase de construcción de la ciudad

\section{NOTA}

La Revisión del Plan General de Ordenación y el Plan Especial deProtección y Rehabilitación de la Ciudad Histórica de Santiago de Compostela han sido elaborados por la Oficina de Planeamiento, S.A. El equipo de dirección del Plan General estuvo formado por los arquitectos Juan Luis Dalda Escudero y Alfonso Díaz Revilla y el economista Anxel Viña Carregal interviniendo como consultores asesores los arquitectos Nuno Rodrigues Portas y Manoel Fernándes de Sá. El equipo de dirección del Plan Especial estuvo formado por los mismos técnicos, con los arquitectos Josef Paul Kleihues y Enrique
Bardají. El Plan Especial de la Ciudad Histórica ha contado con aportaciones $\mathrm{y} / \mathrm{o}$ ha incorporado proyectos de los arquitectos Rafael Baltar Tojo, José Antonio Bartolomé y Carlos Almuiña, Mirko Baum, Alfonso Díaz Revilla, José Díaz Sotelo, Manuel Gallego Jorreto, Celestino García Braña, Mar García García, Giorgio Grassi, John Hejduk, Josef Paul Kleihues, Vittorio Magnano Lampugnani, Carlos Meijide Calvo, Alberto Noguerol del Río y Pilar Díez Vázquez, Felipe Peña Pereda, José Luis Pérez Franco, César Portela Férnandez-Jardón, Helio Piñón y Albert Viaplana, Álvaro Siza Vieira e Isabel Aguirre Urcola.

\section{publicaciones del IETCC/CSIC}

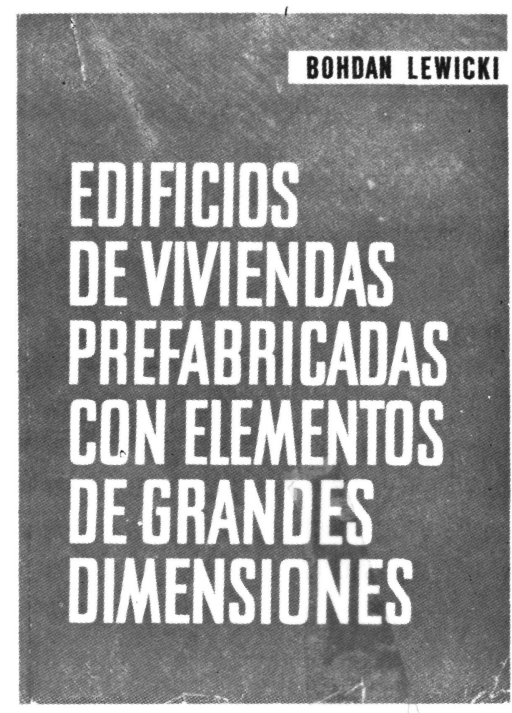

Bohdan Lewicki

Este libro trata de los problemas relativos a la construcción de los edificios de viviendas o publicos realizados con elementos prefabricados de grandes dimensiones. Se han estudiado los problemas de arriostramiento, asi como los que plantea la resistencia de los elementos y de la estructura; se han examinado las cuestiones de orden higrotérmico, acústico y de resistencia al fuego; también se ha profundizado en el estudio de la estanquidad de los muros exteriores y de las juntas.

La obra incluye numerosas ilustraciones que dan detalles de diversas soluciones, asi como ejemplos de cálculo, tablas de valores numéricos, diagramas y ábacos.

Un volumen encuadernado en tela, de $24 \times 17 \mathrm{~cm}$, compuesto de 616 págs
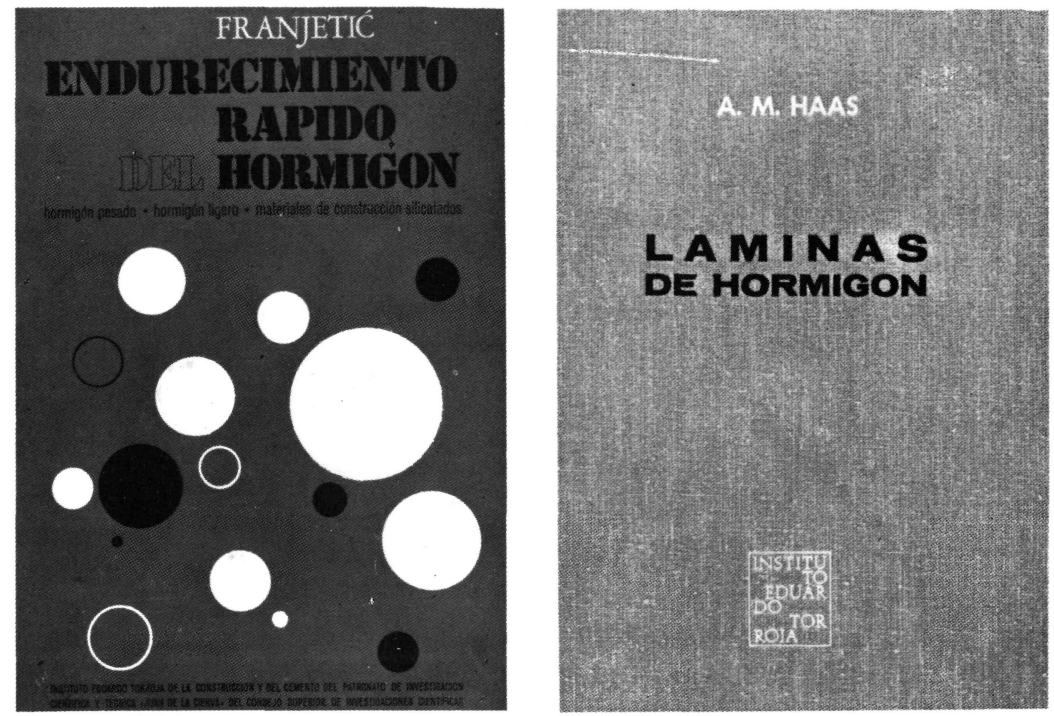

Zorislav Franjetić

En la obra de Franjetić se expone de una forma minuciosa, ordenada y sistemática, todo un cuerpo de doctrina que reúne el conocimiento actual sobre el endurecimiento rápido del hormigón. Parte el autor de los rápido del hormigón. Parte el autor de los
principios básicos y llega a las últimas consecuencias y realidades técnicas y economicas.

Es una obra de consulta, tanto para el investigador sobre la materia, como para el proyectista y el realizador y montador de plantas e instalaciones y equipos de curado y endurecimiento rápido.

Un volumen encuadernado en cartóné, de $17 \times 24,5 \mathrm{~cm}$, compuesto de 385 págs. 110 figuras y 10 tablas.

\section{A. M. Haas}

Al escribir este libro el autor intentó poner a disposición de los estudiantes y de los ingenieros unos conocimientos prácticos, adecuados para servir de guia en el diseño y construcción de láminas delgadas de hormigón.

El autor está convencido de que el éxito en el diseño de una lámina exige, por parte del proyectista, un examen de las tres fases por las que pasa la materialización de la lámina: las que pasa la materialización de la lámina:
el diseño, el análisis estructural y la construcción de la estructura.

Un volumen encuadernado en tela, de $17 \times 24,5 \mathrm{~cm}$, compuesto de 420 págs., 141 figuras, 22 fotografias y 6 tablas. 